\title{
Gradhiva
}

\section{Jeanne Favret-Saada, Désorceler}

Paris, Éditions de l'Olivier, coll. « Penser/Rêver », 2009, 172 p.

\section{Arnaud Esquerre}

\section{OpenEdition}

\section{Journals}

Édition électronique

URL : http://journals.openedition.org/gradhiva/1782

DOI : 10.4000/gradhiva.1782

ISSN : 1760-849X

Éditeur

Musée du quai Branly Jacques Chirac

\section{Édition imprimée}

Date de publication : 19 mai 2010

Pagination : 236-237

ISBN : 978-2-35744-025-8

ISSN : 0764-8928

Référence électronique

Arnaud Esquerre, « Jeanne Favret-Saada, Désorceler », Gradhiva [En ligne], 11 | 2010, mis en ligne le 09 mai 2010, consulté le 10 décembre 2020. URL : http://journals.openedition.org/gradhiva/1782 ; DOI : https://doi.org/10.4000/gradhiva.1782

Ce document a été généré automatiquement le 10 décembre 2020.

(c) musée du quai Branly 


\section{Jeanne Favret-Saada, Désorceler}

Paris, Éditions de l'olivier, coll. « Penser/Rêver », 2009, 172 p.

\section{Arnaud Esquerre}

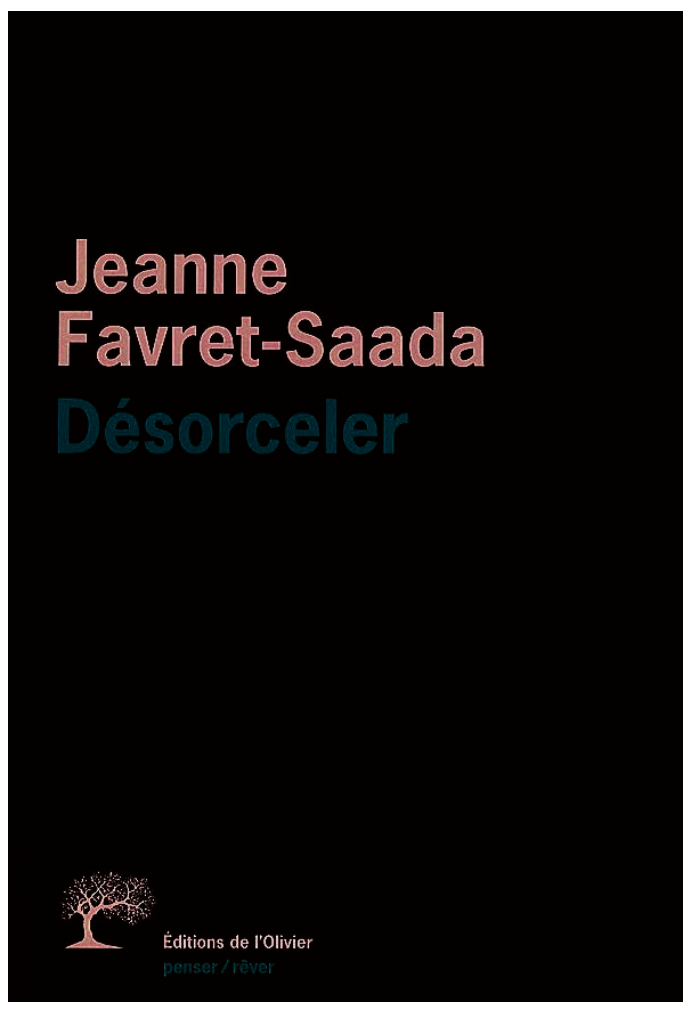

1 Il y a dans tout terrain ou matériaux accumulés par un chercheur en sciences sociales une inintelligibilité qui ne se dissipe que progressivement et longtemps après que celuici a commencé à y réfléchir. Rares sont cependant les chercheurs qui le reconnaissent, et qui ont l'énergie et le temps d'y revenir pour éclairer sous un jour nouveau un sujet dont on pensait, à tort, avoir fait le tour. En 1977, Jeanne Favret-Saada a publié un ouvrage devenu un classique des sciences sociales, Les Mots, la Mort, les Sorts, dans lequel elle analysait la sorcellerie pratiquée par des paysans en Mayenne (suivi de son enquête de terrain, en 1981, dans Corps pour corps, coécrit avec Josée Contreras). Elle y avait alors 
annoncé un second volume consacré au désorcèlement. Elle ignorait à cette époque, comme elle l'avoue, qu'elle n'avait encore "effectué aucun parcours intellectuel à travers le désorcèlement» (p.14). Trente-deux ans plus tard paraît Désorceler (Gallimard 1981), point d'arrivée d'un chemin enfin tracé et parcouru. En cinq chapitres, l'anthropologue a réécrit dix textes publiés, avec Josée Contreras ou seule, entre 1983 et 1991. Même pour ceux qui auraient déjà lu ceux-ci, la surprise reste grande. Articulés les uns aux autres, les textes se lisent différemment, comme on ne regarde pas de la même manière un tableau pris isolément ou environné par d'autres œuvres d'art. Ces textes ont été complétés notamment d'un prélude et d'illustrations commentées et tirées du jeu de tarots de $\mathrm{M}^{\text {elle }}$ Lenormand.

Dans une préface à un ouvrage de Marcelle Bouteiller, Claude Lévi-Strauss avait déclaré que " la sorcellerie étant stérile et non susceptible de progrès ", ceux qui la pratiquent continuent aujourd'hui "à penser comme on a toujours pensé », c'est-à-dire depuis "sans doute des millénaires » (Préface, in Marcelle Bouteiller, Sorciers et jeteurs de sorts, Paris, Plon 1958). De leur côté, les anthropologues anglo-américains niaient la possibilité d'une sorcellerie rurale en Europe dans la seconde partie $d u x^{e}$ siècle (p. 149). Or la sorcellerie, nous montre Jeanne Favret-Saada, existe dans les années 1970 dans le bocage normand, alors qu'elle était supposée ne plus exister. Elle a évolué en s'adaptant depuis le XIX ${ }^{\mathrm{e}}$ siècle, alors qu'elle était censée être figée. Et elle est attachée à un tissu social particulier, celui de la paysannerie bocaine, qui s'est modifié profondément depuis les années 1980 si bien qu'en l'absence de nouveaux travaux l'on ne sait pas au début du xxI ${ }^{\mathrm{e}}$ siècle si, en France, la sorcellerie existe encore ni sous quelle forme.

3 Les Mots, la Mort, les Sorts se concluaient par un exposé du système conceptuel qui soustend la représentation que les ensorcelés se font de ce dans quoi ils sont pris (1977). Dans une "crise de sorcellerie », un sorcier entreprend d'attirer à lui la force vitale d'un individu quelconque. Ce dernier n'a pas d'autre issue, pour réagir et éviter d'être ruiné et de mourir, que de faire appel à un désorceleur. Celui-ci oppose à l'agresseur une force magique telle qu'elle contraint le sorcier à restituer à l'ensorcelé la quantité de force vitale dérobée. Comment cela est-il possible? Par la pratique d'une thérapie, selon Jeanne Favret-Saada. Dans le sillage de ce travail, Désorceler propose de réaliser une anthropologie des thérapies, pensées à partir de la sorcellerie. Dans le bocage, le savoir sur le désorcèlement et le savoir-faire rituel sont dissociés. Le désorceleur est censé compter sur la seule efficacité de l'acte rituel, et l'ordre du « faire » («ça y fait ») ne pas nécessiter de justifications théoriques. Mais autre chose se passe en réalité. L'auteur identifie deux sortes de récits oraux: les premiers, "exemplaires», sont destinés à persuader l'auditeur par l'exemple; et les seconds, «incitatifs", invitent l'auditeur à s'engager dans le désorcèlement. Un récit «exemplaire» met en scène l'efficacité de la sorcellerie (des malheurs dont est victime l'ensorcelé au rituel du désorceleur frappant le sorcier). Un récit «incitatif» montre la relation entre un désorceleur et les ensorcelés, et vise à expulser ceux-ci de leur place de victimes ; c'est cette relation que l'anthropologue qualifie de «thérapeutique » (p. 51).

Jeanne Favret-Saada analyse le travail de $\mathrm{M}^{\mathrm{me}}$ Flora, une désorceleuse dont elle a été la cliente et le témoin pendant deux ans. Contrairement à ses collègues, cette désorceleuse, impotente, ne pouvait se rendre dans les fermes et désorcelait à domicile, en s'aidant de la cartomancie. Comment une désorceleuse s'y prend-elle pour dynamiser ceux qui viennent la voir en étant abattus par une série de catastrophes? 
Les traités français de pratique divinatoire mettent l'accent sur le choix du jeu de cartes, leur mode de tirage et leurs significations, comme si ces dernières contenaient par avance l'avenir du consultant. Or le travail de la voyante consiste tout d'abord «à construire, par approximations successives, des énoncés recevables sur la situation particulière du client » (p. 92). Dans la première phase d'une séance, la désorceleuse s'appuie sur deux lots de cartes de jeu de piquet, l'un lui permettant de tenir un discours sur le bien et le mal, l'autre lui servant à identifier les problèmes de la vie quotidienne des consultants.

Alors que la signification donnée aux cartes rouges est celle du bien et renvoie aux ensorcelés, la signification donnée aux cartes noires est celle du mal et renvoie aux sorciers. Lorsque la femme vient sans époux, la désorceleuse interprète une carte rouge, la dame de carreau, comme étant la sorcière, et une carte noire, la dame de pique, comme étant l'ensorcelée, et elle commente alors à l'attention de la femme venue la consulter: "Dans l'avenir, vous deviendrez veuve », ce qui, d'après Jeanne Favret-Saada, signifie : "Vous, vous êtes forte, pas comme votre faiblard de mari, et c'est pourquoi dans un avenir lointain vous deviendrez veuve, c'est-à-dire que vous survivrez. » (p. 90) Le dispositif, incluant cette phrase, redonne de l'énergie à la femme venue en consultation. Josée Contreras et Jeanne Favret-Saada ont nommé ce dispositif, destiné à compromettre à leur insu les consultants avec le mal et le sorcier, "l'embrayeur de violence» en référence aux embrayeurs du discours étudiés par Roman Jakobson (Essais de linguistique générale. Paris, éditions de Minuit 1963). Les clients acceptent (sans pour autant le reconnaitre) de souhaiter la souffrance et la mort du sorcier. La désorceleuse utilise alors, dans la deuxième phase d'une séance, un jeu de tarots dont les figures fortes, combinées à l'éloquence de $\mathrm{M}^{\mathrm{me}}$ Flora, emportent la conviction des consultants. Jeanne Favret-Saada rapporte, à partir des reproductions de vingt-cinq motifs de ces tarots, les interprétations délivrées par la désorceleuse montrant le sorcier, décrit souvent comme un prédateur, confronté aux ensorcelés, ses minuscules victimes désarmées.

6 En fin de séance, la désorceleuse prescrit aux consultants un programme d'actions qui leur permettront de retrouver l'initiative, et qui consistent notamment en une multitude de petites tâches (parmi lesquelles réaliser des sachets en toile rouge et les garnir avec des ingrédients spécifiques ou encore disposer sous le lit des planchettes hérissées de clous). Or le désorcèlement, montre l'anthropologue, « ne peut atteindre son objectif qu'en faisant jouer les rapports sociaux entre les sexes » (p. 125). L'homme, qui reconnaît implicitement son impuissance à préserver le potentiel bioéconomique de son exploitation, s'engage dans une théorie des sorts en minimisant son adhésion et en soulignant que sa femme y « croit » davantage que lui. Car, dans un premier temps, la thérapie sorcellaire guérit la femme de façon directe puis, dans un second temps, le travail de l'épouse guérit le mari (p. 132).

7 Tout au long de son ouvrage, Jeanne Favret-Saada analyse la thérapie sorcellaire en s'appuyant sur une conception de l'être humain à rebours des idées reçues circulant souvent dans les sciences sociales comme des "évidences", alors qu'elles sont des constructions que l'anthropologue estime inadaptées ou incorrectes. Elle avait proposé, dans une critique de Max Weber («Weber, les émotions et la religion», Terrain 22 : 93-108, 1994), de ne pas raisonner en s'appuyant sur le couple de la Raison et de l'Émotion, et elle lui a substitué un paradigme unitaire, qui allie indissociablement l'affect et les formes logiques. Dans Désorceler, partant de son expérience qui fait place 
«à la communication non verbale, non intentionnelle, involontaire, au surgissement et au libre jeu d'affects dépourvus de représentations ", elle considère qu'un être humain est constitué d'une "opacité ", notion "vieille comme la tragédie » et qui peut être nommée «inconscient» ou encore autrement (p.161). Cette opacité est évidemment partagée : l'anthropologue ne la rencontre pas seulement chez les autres, mais il est opaque à lui-même. Pour éclairer et dissiper, autant que possible, cette opacité des relations humaines, l'auteur propose de renouveler les méthodes en cours. Mais alors que Les Mots, la Mort, les Sorts et Corps pour corps mettaient en œuvre la méthode de Jeanne Favret-Saada par des récits à la première personne, Désorceler présente les résultats de l'enquête menée en rompant avec la narration et en adoptant un ton analytique. Être présent sur le terrain, conclut l'anthropologue, induit d'y occuper une place et d'en tirer les conséquences : accepter de participer et d'être affecté, c'est-àdire d'être altéré par l'expérience vécue.

Chaque objet social nécessite, sans doute, une méthode plus particulièrement adaptée pour y accéder, le restituer et le comprendre, et les chercheurs peuvent être affectés avec des intensités plus ou moins grandes, notamment parce que chaque terrain est doté de propriétés singulières. Et certains terrains peuvent peut-être donner mieux que d'autres l'illusion qu'une observation participante est possible. Mais pour qui voudra entreprendre une anthropologie des thérapies en suivant la voie ouverte par Jeanne Favret-Saada, il faudra accepter le risque d'être profondément affecté. Une autre conséquence importante impliquée par le paradigme unitaire de l'intériorité humaine concerne le rapport aux images analysé par Désorceler. Selon une idée répandue, seules les personnes les plus cérébrales résisteraient au passage automatique de la vue à l'empathie et à l'implication, faisant triompher la raison sur la spontanéité des sentiments provoqués par la perception (voir Freedberg 1998 [1989], Le Pouvoir des images. Paris, Gérard Monfort). Cette conception légitime souvent la soustraction des images aux regards de ceux qui n'auraient pas la force ou la volonté d'y résister (parce qu'ils seraient des êtres « faibles »). Or une analyse des effets des cartes de $\mathrm{M}^{\text {me }}$ Flora se fondant sur une dissociation entre raison et émotion n'aurait pas pu rendre compte de ceux-ci. Les images n'ont pas d'effet par elles-mêmes; elles n'ont d'effets que ceux produits par l'agencement dans lequel elles sont insérées. Cependant, ce n'est que parce que Jeanne Favret-Saada postule un paradigme unitaire de l'intériorité humaine qu'elle parvient à saisir les effets si variés des cartes, et en particulier cet effet thérapeutique qui n'est pas le moindre: le déminage de l'anxiété de ceux qui les regardent.

\section{AUTEUR}

\section{ARNAUD ESQUERRE}

a_esquerre@hotmail.com 